\title{
Erratum to: LAPTM4B-35 is a novel prognostic factor for glioblastoma
}

\author{
Xiaoshu Dong ${ }^{1} \cdot$ Kaoru Tamura $^{1}$ (D) Daisuke Kobayashi ${ }^{2}$ ' Noboru Ando ${ }^{2}$. \\ Kazutaka Sumita $^{1} \cdot$ Taketoshi Maehara $^{1}$
}

Published online: 9 March 2017

(C) Springer Science+Business Media New York 2017

\section{Erratum to: J Neurooncol \\ DOI 10.1007/s11060-017-2369-0}

The original article contains the following errors:

1. The first author's name was incorrect (Xiaoshud Dong); it is correct in this erratum (Xiaoshu Dong).

2. In Fig. 2, the survival curves of A and B were interchanged. In addition, the number of patients specified in the legend of Fig. 2 was incorrect. The number of patients with high LAPTM4B-35 expression should have read 23 (instead of 22) and the number of patients with low LAPTM4B-35 expression should have read 16 (instead of 17). The correct Fig. 2 and caption are printed below.

3. In the Table 1, the number of LAPTM4B low patients should have read 16 (instead of 12).

4. In the Table 1, the number of LAPTM4B high patients should have read 23 (instead of 27).

5. In the Supplementary Table 1, the number of gross total resections in grade IV patients should have read $12(27.9 \%)$, instead of $21(48.8 \%)$.
The online version of the original article can be found under doi:10.1007/s11060-017-2369-0.

Kaoru Tamura

kaoru-tmd@umin.ac.jp

1 Department of Neurosurgery, Tokyo Medical and Dental University, 1-5-45 Yushima, Bunkyo-ku, Tokyo 113-8510, Japan

2 Department of Pathology, Tokyo Medical and Dental University, 1-5-45 Yushima, Bunkyo-ku, Tokyo 113-8510, Japan 


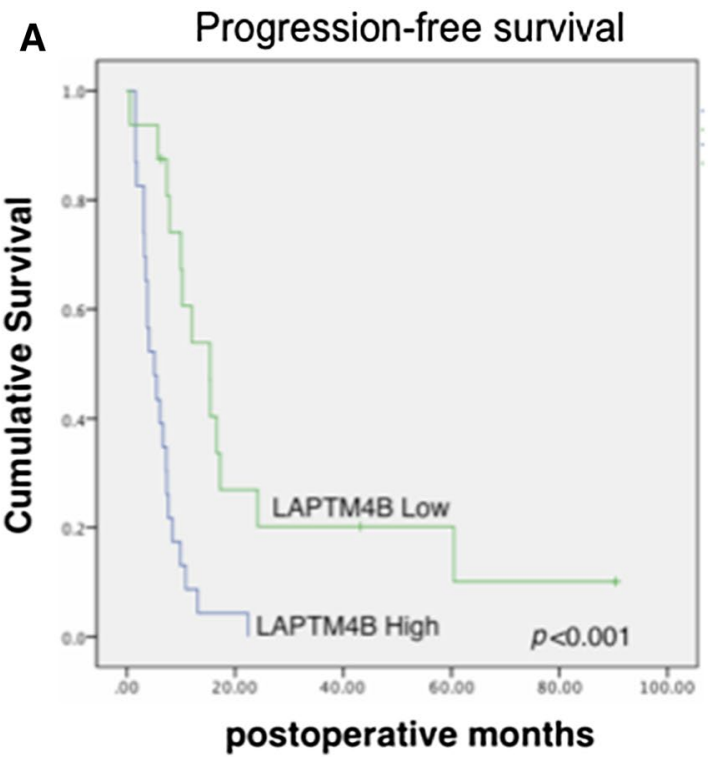

Fig. 2 Kaplan-Meier curves for progression-free survival and overall survival in 39 GBM patients according to LAPTM4B-35 expression. a The median progression-free survival of GBM patients with high LAPTM4B-35 expression $(n=23)$ was $5.13 \pm 4.72$ months, significantly shorter than that of patients with low LAPTM4B-35

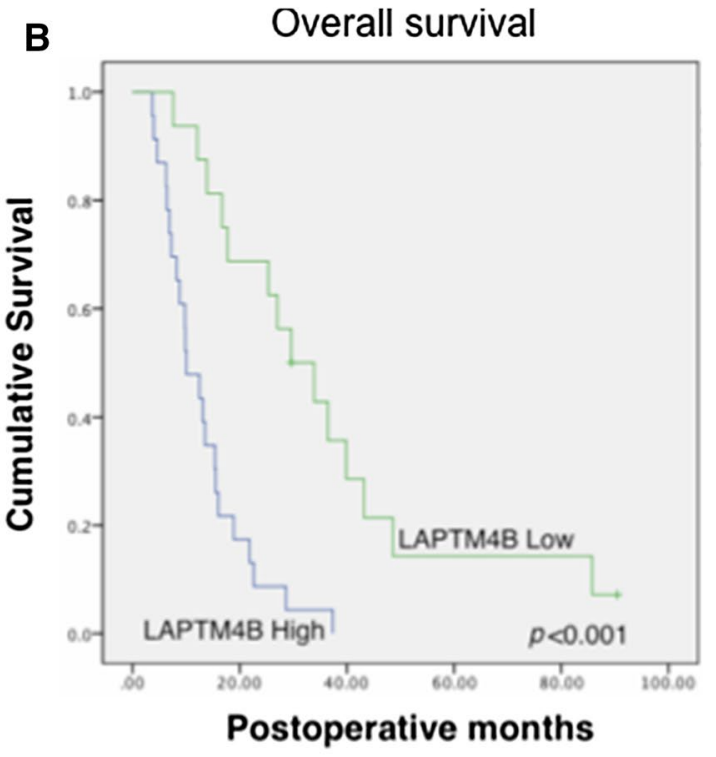

expression $(\mathrm{n}=16,12.0 \pm 23.82$ months, $p<0.001)$. b The median overall survival of GBM patients with high LAPTM4B-35 expression $(n=23)$ was $12.5 \pm 8.34$ months, again significantly shorter than that of patients with low LAPTM4B-35 expression $(\mathrm{n}=16,29.6 \pm 23.7$ months, $p<0.001$ ) 